\title{
Effects of relaxation and stress management training on fibromyalgia symptoms in women
}

\author{
Rasol Roshan $^{1}$, Azadeh Tavoli ${ }^{2 *}$, Neda Sedighimornani ${ }^{3}$, Zahra Goljani $^{1}$ and Seyed Shamsia Shariatpanahi ${ }^{4}$ \\ ${ }^{1}$ Department of Psychology, School of Humanities, Shahed University, Tehran, Iran \\ ${ }^{2}$ Department of Psychology, Faculty of Educational Sciences and Psychology, Alzahra University, Tehran, Iran \\ ${ }^{3}$ Department of Psychology, Faculty of Humanities and Social Sciences, University of Bath, Bath, UK \\ ${ }^{4}$ Department of Romathology, Faculty of Medicine, Shahed University, Tehran, Iran
}

\begin{abstract}
This study compared the effectiveness of relaxation training and stress management training in treating anxiety, depression, stress, and pain in women with fibromyalgia. Twenty-two women with fibromyalgia participated in this randomized, controlled trial. They were assigned to stress management $(n=7)$, relaxation training $(n=7)$, and control ( $n=8)$ groups. All participants completed the Depression, Anxiety, and Stress Scale (DASS-42) and the Visual Analog Scale (VAS) for pain. The stress management group participated in eight weekly two-hour sessions of a stress management program; the relaxation group participated in eight weekly sessions of relaxation training. Then, participants completed the DASS-42 and VAS again. Analyses of Covariance (ANCOVA) and post hoc analyses were used to compare the participants' pre- and post-therapy responses and the two treatment types. Both relaxation training and stress management training were found to be effective in treating patients' anxiety $(P=0.005)$, depression $(P=0.006)$, stress $(P=0.002)$, and pain $(P=0.001)$. Post hoc analyses indicated that participants in the treatment groups showed improvement compared with those in the control group (all $P$ values $<0.01$ ). However, there was no significant difference in effectiveness between the relaxation training and the stress management training. The relaxation training and stress management training both significantly improved women's fibromyalgia symptoms (depression, stress, anxiety, and pain).
\end{abstract}

Keywords: fibromyalgia, relaxation, stress management.

\section{Introduction}

Fibromyalgia is a medical musculoskeletal condition that is difficult to diagnose or even to fully describe. There is much controversy about identifying it; its classification criteria are constantly being updated. However, rheumatologists generally consider fibromyalgia to be a primary condition that may coexist with other conditions. Its hallmarks include widespread body pain as well as fatigue, nonrestorative sleep, a decreased threshold for pain, and cognitive deficits $[1,2]$. In Iran, the prevalence rate of fibromyalgia is estimated to be $4 \%$ to $5 \%$ [3, 4], which is comparable with its prevalence rate in other countries $[3,5]$. In general, women are affected more than men $[3,5]$.

Fibromyalgia is associated with negativity, mood disturbance, depression, and anxiety [6-9]. It is often preceded by childhood trauma or stressful life events [2]. In the majority of patients, quality of life, productivity, and functional abilities are noticeably reduced as patients usually experience significant lethargy, pain, and otherwise debilitating disabilities $[5,10]$. Fibromyalgia is therefore considered one of the most complex and costly diseases for patients and healthcare providers [11, 12]. More effective symptom management and treatment options are needed to help sufferers and offset the costs of this condition [13].

Possible treatment options include pharmacological and non-pharmacological interventions [13, 14]. Medication therapies, however, tend to be only mildly or moderately effective for managing fibromyalgia [15]. As a result, researchers are now focusing more on non-pharmacologic treatments or on a combination of pharmacological and non-pharmacologic treatments [13]. Non-pharmacologic interventions include physical activities such as aerobic or cardiovascular exercise, educational or psychoeducational programs, cognitive-behavioural therapy, alternative therapies such as acupuncture and Tai Chi, and various relaxation techniques [16-21]. 
Previous research has indicated that psychological interventions are highly beneficial for patients with fibromyalgia. For example, Vázquez-Rivera et al. [18] demonstrated that a brief cognitive behavioural therapy (CBT) intervention was effective in reducing emotional distress in women suffering from fibromyalgia. In their study, 34 female outpatients participated in a CBT program for five to six weeks. They were assessed for depression, anxiety, coping strategies, physical functioning, and somatization before and after the intervention. Brief CBT group therapy sessions were found to be effective in reducing the patients' emotional distress. Similarly, a meta-analysis of studies employing cognitive behavioural therapy for fibromyalgia patients showed that CBT-based programs enhance patients' ability to cope with pain, decrease depression, and improve healthcare-seeking behaviours [22].

King et al. [23] compared the efficacy of combined therapies and individual intervention methods in treating female fibromyalgia patients. The therapies they tested were a supervised aerobic exercise, a selfmanagement education program, and a combination of the exercise and education programs. Those patients treated with the combination of the exercise and education programs (and who complied with the treatment protocol) benefited the most. It seems that educational programs [24-26] in addition to relaxation, stress management training, and meditation-based programs [27-31] can have immediate and often sustained benefits for fibromyalgia patients [25, 26].

The current study aimed to confirm and extend previous findings supporting the use of psychological interventions for managing fibromyalgia. Specifically, the efficacy of relaxation and stress management therapies was tested for the treatment of Iranian women's anxiety, stress, depression, and pain. In this study, the effects of relaxation and stress management strategies on fibromyalgia symptoms were also compared.

\section{Methods}

\section{Participants}

This randomized control trial tested the participants' pre- and post-intervention symptoms. Participants were informed about the study, and those willing to participate were randomly assigned to the intervention groups or the control treatment. Intervention lasted for eight weeks. Patients were examined before any therapies were administered (to establish a baseline) and at the end of the study. The examinations and interventions were completed by a clinical psychologist.

Participants were all patients with fibromyalgia attending the teaching hospital of Shahed University of Medical Sciences (Mostafa Khomeini Hospital) in Tehran, Iran for treatment. They were assessed by a rheumatologist using the American College of Rheumatology (ACR) criteria for diagnosing fibromyalgia [1]. The selected patients met the following criteria:

1. They experienced widespread pain lasting for at least three months in 11 of the 18 specific tender points identified by the ACR [32];

2. They exhibited no signs of a current psychotic disorder or substance abuse;

3. They exhibited no signs of an organic mental disorder;

4. They were able to read and write;

5. They had not received similar psychotherapy treatments previously;

6. They were aged 25 to 70 years.

Participants who met these criteria were then informed of the study's methods and goals. Thirty patients were willing to participate; eight of them were excluded from the final results, because they were absent for more than two treatment sessions. The twenty-two remaining patients were randomly assigned to the relaxation group $(n=7)$, the stress management group $(n=7)$, or the control group $(n=8)$.

The control group comprised eight patients previously diagnosed with fibromyalgia who were attending Mostafa Khomeini Hospital for a standard visit. They were all female (mean age $=57.13 \pm 7.37$ ) and literate (mean years of education $=9.88 \pm 4.25$ ). The study was conducted in compliance with the ethical standards of the regional research committee. Participation was voluntary, and informed consent was obtained from each participant. Patient anonymity was preserved.

\section{Statistical analysis}

Data were analyzed using the statistical software program SPSS, v. 21.0.0. Analyses of Covariance (ANCOVAs) and the post hoc Bonferroni test were used for group comparisons. The results of the analyses were adjusted for multiple comparisons at a 0.01 interval confidence (Bonferroni correction). A $P$-value of $<0.05$ was considered statistically significant for the purposes of this study. 


\section{Procedures}

\section{Relaxation therapy}

The relaxation technique used for this study was based on the approach of Bernstein and Borkovec (1973) [33], which is similar to that of Wolpe and Jacobson [34]. In this method, clients are trained to understand the purposes of the exercises they complete during their progressive relaxation sessions. They are asked to pay attention to a particular muscle group and concentrate on the sensations felt in those muscles. Then, they are invited to tense the muscle group for five seconds before relaxing it again. After relaxing the muscle group, they are instructed to focus again on the sensations in those muscles. This tensing and relaxing exercise is subsequently completed on a different muscle group. Bernstein and Borkovec modified Jacobson's original method in that the therapist does not ask participants to differentiate between different degrees of tension. Instead, patients are instructed to either tense or relax a muscle group thoroughly [33]. In the present study, patients $(n=7)$ participated in eight one-hour sessions of this group relaxation training.

\section{Stress management training}

Stress management training is a psychoeducational program for dealing with stress. Patients are taught to become aware of their appraisal of stressful events. By doing so, they can learn how to better cope with the negative feelings that result from these events. Stress management training utilizes a variety of techniques, including self-management skills, behaviour modification, CBT, emotional expression, and physical activity [30].

In this study, the stress management program was designed to meet the needs of the fibromyalgia patients by focusing on the self-management of pain and emotional distress [30]. The patients in this group ( $\mathrm{n}=$ 7) participated in eight weekly two-hour sessions of group stress management training. The sessions were based on the stress management intervention model and included discussions of the following elements:

1. the meaning of stress and its signs (first session);

2. the relationship between stress and fibromyalgia symptoms (second session);

3. managing and coping with stress (third session);

4. problem solving (fourth session);

5. time management (fifth session);

6. anger management (sixth session);

7. healthy lifestyles (seventh session), and

8. advice for stress management (eighth session).
A psychologist with comprehensive experience in conducting psychotherapy and trained in relaxation and stress management training conducted these sessions.

\section{Symptom measures}

Visual Analog Scale (VAS). The VAS has been shown to be a valid and reliable scale for assessing intensity of pain in rheumatology patients. Specifically, it is often used to assess mental parameters in clinical trials of fibromyalgia patients [35]. It has been proven to be a sound instrument for the quantitative assessment of acute pain and the detection of clinically important changes in such pain in Iran [36].

The VAS measures pain on a continuous scale, using either a vertical or a horizontal line. Respondents in this study were asked to indicate their level of pain intensity by marking a point on a 10-point horizontal VAS line. The score was defined by measuring the distance between the line's endpoint and the patient's mark on the line; higher scores indicate higher pain intensity. Respondent burden is very low for this measure, as the test takes less than one minute.

Depression, Anxiety, Stress Scale (DASS-42). Depression, anxiety, and stress were measured using the DASS-42 [37], a 42-item self-inventory that assesses three axes of a patient's wellbeing: depression, anxiety, and stress (in the last seven days). This measure separates physical anxiety (fear symptomatology) from mental stress (nervous tension and nervous energy) into two distinct factors. This scale has been shown to be a reliable and in-depth measure of patient symptoms [38].

The DASS anxiety scale correlates significantly with the Beck Anxiety Inventory (BAI) $(r=0.80)$ and the DASS depression scale correlates significantly with the Beck Depression Scale (BDI) ( $r=0.74)$. This study employed the Iranian version of the DASS-42, which has previously been shown to be a valid method for quantifying patients' symptoms in Iran [39].

\section{Results}

The findings demonstrated that there were no significant differences between participants in the three groups (stress management, relaxation, and control) in terms of age or education. Table 1 shows the demographic breakdown of the study participants.

In Table 2, the means and standard deviations for the two symptom scales are presented. Before intervention, the three groups were not statistically different in terms of pain, anxiety, stress, or depression. 
Relaxation versus stress management training for fibromyalgia

Table 1. Demographic variables of participants

\begin{tabular}{|c|c|c|c|c|c|c|c|}
\hline Conditions & $\begin{array}{c}\text { Condition } 1 \\
\text { Relaxation Group } \\
(\mathbf{N}=7)\end{array}$ & $\begin{array}{c}\text { Condition } 2 \\
\text { Stress management group } \\
(\mathrm{N}=7) \\
\text { Mean (SD) }\end{array}$ & $\begin{array}{c}\text { Condition } 3 \\
\text { Control group } \\
(\mathbf{N}=\mathbf{8})\end{array}$ & $\begin{array}{l}\text { Min - } \\
\text { Max }\end{array}$ & Range & $\mathbf{F}$ & $\mathbf{p}$ \\
\hline Age (years) & $53.8(4.06)$ & $56.8(7.1)$ & $57.1(7.3)$ & $42-69$ & 27 & 0.65 & 0.52 \\
\hline Education (years) & $12.8(3.2)$ & $11.7(1.8)$ & $9.9(4.2)$ & $5-19$ & 14 & 1.53 & 0.24 \\
\hline
\end{tabular}

Note: $\mathrm{SD}=$ Standard Deviation; $* * P<0.01 ; * P<0.05$, all patients were female

Table 2. Means, standard deviations, and ANCOVA (s) showing the significance effect of condition on pain, anxiety, depression, and stress after controlling for the effect of pre-test scores

\begin{tabular}{|c|c|c|c|c|c|c|c|c|c|c|}
\hline & \multicolumn{2}{|c|}{$\begin{array}{c}\text { Relaxation group } \\
(\mathrm{N}=7)\end{array}$} & \multicolumn{2}{|c|}{$\begin{array}{l}\text { Stress management } \\
\text { group }(N=7)\end{array}$} & \multicolumn{2}{|c|}{$\begin{array}{c}\text { Control group } \\
(\mathrm{N}=8)\end{array}$} & \multirow{3}{*}{$\mathbf{F}$} & \multirow{3}{*}{$\mathbf{P}$} & \multirow{3}{*}{$\begin{array}{l}\text { Partial } \\
\quad \eta 2\end{array}$} & \multirow{3}{*}{$\begin{array}{c}\text { Observed } \\
\text { Power }\end{array}$} \\
\hline & Pre-test & Post-test & & Post-test & Pre-test & Post- test & & & & \\
\hline \multicolumn{7}{|c|}{ Mean (SD) } & & & & \\
\hline Pain & $6.43(1.6)$ & $5(1.15)$ & $6.7(1.6)$ & $3.7(2.3)$ & $7(1.7)$ & $7.50(1.4)$ & 11.59 & $0.001 * *$ & 0.30 & 0.75 \\
\hline Anxiety & $16.8(6.6)$ & $8(6.8)$ & $\begin{array}{l}23.7 \\
(6.4)\end{array}$ & $\begin{array}{c}12.1 \\
(10.5)\end{array}$ & $\begin{array}{l}24.5 \\
(5.1)\end{array}$ & $23.7(4.7)$ & 7.26 & $0.005^{* *}$ & 0.44 & 0.88 \\
\hline Depression & $16.5(6.8)$ & $6.5(7)$ & $\begin{array}{l}24.3 \\
(4.6)\end{array}$ & $13(12.6)$ & $\begin{array}{l}23.2 \\
(6.4)\end{array}$ & $24(5.2)$ & 6.95 & $0.006^{* *}$ & 0.43 & 0.87 \\
\hline Stress & $\begin{array}{l}16.86 \\
(6.5)\end{array}$ & $8(6.8)$ & $\begin{array}{c}23.71 \\
(6.4)\end{array}$ & $\begin{array}{l}12.14 \\
(10.5)\end{array}$ & $\begin{array}{l}24.50 \\
(5)\end{array}$ & $\begin{array}{c}24.75 \\
(4.7)\end{array}$ & 9.04 & $0.002 * *$ & 0.50 & 0.94 \\
\hline
\end{tabular}

Note: $* * P<0.01 ; * P<0.05 ; \mathrm{SD}=$ Standard Deviation

However, patients in both treatment groups showed significant improvement in every measure: pain level $(\mathrm{F}(2,18)=11.59, P=0.001)$, anxiety $(\mathrm{F}(2,18)=7.26$, $P=0.005)$, depression $(\mathrm{F}(2,18)=6.95, P=0.006)$, and stress $(\mathrm{F}(2,18)=9.04, P=0.002)$. The significance of these effects was calculated after controlling for the pre-test symptom scores.

Post hoc Bonferroni tests were also conducted to compare the control group with the two treatment groups. Participants in the treatment groups showed significant improvements in terms of pain, anxiety, stress, and depression compared with the control group. However, there was no significant difference between the two treatment groups in terms of depression $(P>0.05)$, pain severity $(P>0.05)$, anxiety $(P>0.05)$, or stress $(P>0.05)$. That is, while treatment significantly improved patient symptoms (as compared to no treatment), the two treatment types proved equally effective.

\section{Discussion}

This study assessed the benefits of relaxation and stress management training in patients with fibromyalgia. Twenty-two women were assigned to the relaxation group, the stress management group, or the control group. After eight training sessions, participants who had attended either training program demonstrated considerable improvements in terms of pain, anxiety, stress, and depression compared with the control group.

These results are compatible with the previous findings reported by Fors et al. [40], Menzies et al. [41], Anderson et al. [42], Castel et al. [43], Kaplan et al. [27], and Wigers et al. [44]. Menzies and Kim [41], for example, showed that ten weeks of visual imagerydriven relaxation sessions enhanced fibromyalgia patients' abilities to manage pain. Similarly, Wigers, Stiles, and Vogel [44] tested the effectiveness of 14 weeks of stress management treatment or aerobic exercise on patients with fibromyalgia. They found that both stress management and aerobic exercise had positive effects on managing pain, depression, and a lack of energy.

As the VAS scale results revealed in this study, the relaxation technique and the stress management training reduced the patients' levels of pain. However, participants' pain levels were still moderately high after treatment (any score on the VAS equal to or above 4 out of 10 was considered significant). Therefore, future studies may need to consider 
prescribing more therapeutic sessions over a longer period of time or combining therapy techniques for greater effectiveness. Fibromyalgia patients should not have to suffer such chronic, significant pain.

There is increasing agreement about the role of disturbances in central pain processing in fibromyalgia syndrome [45] and the intrinsic links between these disturbances and physical or psychosocial stress. This idea is part of a biopsychosocial model [46]. From a neurohormonal point of view, the stress response is mainly processed by the sympathetic (or locus coeruleus/ norepinephrine (LCNE)) axis, acting in close connection with the hypothalamus-pituitaryadrenal (HPA). These mechanisms are affected by the corticoliberin hormone, which is mainly discharged by the hypothalamus and the amygdala [47].

Most clinicians believe that both physical and emotional stresses are present in fibromyalgia patients [48]. Kivimäki et al. [49] examined the prospective association between occupational stress and the incidence of newly diagnosed fibromyalgia. After adjusting for covariates, the authors found that high workload, having less freedom to make decisions at work, and experiencing bullying at work correlated with patients' emergent fibromyalgia symptoms. It seems that fibromyalgia patients are not able to manage their emotions and everyday conflicts adequately [50], which may explain why they experience a high level of stress at the beginning of their disease. Thus, stress management programs are likely to be beneficial because they may enhance patients' abilities to manage their own symptoms [51].

In accordance with previous findings [27, 41], patients in this study who attended the training programs, perceived a substantial decrease in their levels of anxiety and depression. The findings further indicated that there was no difference between the therapeutic benefits of the relaxation techniques and the stress management training.

Anxiety and depression are two of the psychological symptoms that have been observed in many patients with fibromyalgia. However, fibromyalgia is not a homogeneous disease; it shows varying proportions of comorbid anxiety and depression, depending on the psychosocial characteristics of each patient. While assessing patients with fibromyalgia, widespread pain, as well as the existence of emotional distress, should be considered [2]. Relaxation [52] and stress management training [53] are usually designed to reduce anxiety and stress. It is not surprising, then, that patients in this study found both methods to be effective for reducing their anxiety and depression.

The study design did not allow which aspects of the treatments were responsible for the improvements in the experimental groups to be determined. It may be assumed that providing patients with information about their illness and clear behavioural instructions for fostering self-control are the effective ingredients of the treatments, since these elements have been demonstrated to produce positive results in other studies [54].

Finally, the limitations of the present study should be acknowledged. First, the sample size was small. Therefore, the results should be treated with caution; they may not be generalizable to all fibromyalgia patients, in Iran or elsewhere. Second, the patients in this study suffered mild to moderate disabilities from their disease, which prevented the generalization of the present results to patients with more severe fibromyalgia. The duration of the study was also relatively short. A longer follow-up is required to determine whether or not patients' symptoms returned after completing treatment.

Lastly, the current research included only female patients. Future studies will need to obtain information about male and female patients and their quality of life. It is not yet known whether or not there is a difference between men and women in relation to the success of the treatment programs tested in this study. Furthermore, it is not clear why the two treatments were equally effective. They might have common traits that produce a common sense of self-empowerment and ability to self-manage symptoms of fibromyalgia, or they might reduce stress and anxiety by influencing the body's performance. This is an issue that needs further inquiry.

Future analyses should also consider that some of the techniques learned with a trained professional could be practiced at home. Patients should be encouraged to practice these techniques on their own in a long-term fashion, and the effectiveness of these athome practices should be investigated. To manage a complex disease such as fibromyalgia, it is also suspected that pharmacological and psychological interventions might improve the overall treatment plan.

\section{Conclusion}

Relaxation techniques and stress management training seem to be beneficial for women with fibromyalgia, as was demonstrated by patients' scores on the DASS-42 and the VAS in this study. No difference was observed 
in the effectiveness of these two treatments for the fibromyalgia patients in this study. In the future, the effectiveness of combined therapies should be analyzed. Furthermore, patients should be encouraged to practice techniques learned with trained professionals at home, and researchers should investigate the long-term wellbeing of such patients.

\section{Conflicts of interest}

The authors declare no conflicts of interest.

\section{Acknowledgments}

The authors would like to thank all the patients who participated in the study.

\section{References}

1. Wolfe F, Smythe HA, Yunus MB, Bennett RM, Bombardier C, Goldenberg DL. The American College of Rheumatology 1990 criteria for the classification of fibromyalgia: Report of the multicenter criteria committee. Arthritis Rheum 1990; 33(2): 160-172.

2. Thieme K, Turk DC, Flor $\mathrm{H}$ Comorbid depression and anxiety in fibromyalgia syndrome: relationship to somatic and psychosocial variables. Psychosom Med 2004; 66(6): 837-844. doi: 10.1097/01.psy. 0000146329.63158.40.

3. Hadianfard MJ, Hosseinzadeh Parizi M. A randomized clinical trial of fibromyalgia treatment with acupuncture compared with fluoxetine. Iran Red Crescent Med J 2012; 14(10): 631-40.

4. Banihashemi AT, Davatchi F, Jamshidi AR, Faezi T, Paragomi $\mathrm{P}$, Barghamdi, M. Prevalence of osteoarthritis in rural areas of Iran: a WHO-ILAR COCPORD study. Int J Rheum DS 2014; 17(4): 384-388. doi: 10.1111/ 1756-185x.12312.

5. Jahan F, Nanji K, Qidwai W, Qasim R. Fibromyalgia syndrome: an overview of pathophysiology, diagnosis and management. Oman Med J 2012; 27(3): 192-5. doi: 10.5001/omj. 2012.44.

6. Bateman L, Sarzi-Puttini P, Burbridge CL, Landen JW, Masters ET, Bhadra Brown P, et al. Burden of illness in fibromyalgia patients with comorbid depression. Clin Exp Rheumatol 2016; 34(2 Suppl 96): 106-13.

7. Offenbaecher $\mathrm{M}$, Glatzeder $\mathrm{K}$, Ackenheil M. Self-reported depression, familial history of depression and fibromyalgia (FM), and psychological distress in patients with FM. Rheumatol
1998; 57(8): 94-96. doi: 10. 1007/ s003930050245.

8. Bilgin S, Arslan E, Elmas O, Yildiz S, Colak OH, Bilgin G, et al. Investigation of the relationship between anxiety and heart rate variability in fibromyalgia: A new quantitative approach to evaluate anxiety level in fibromyalgia syndrome. Comput Biol Med 2015; 1(67): 126-35. doi: 10. 1016/j.compbiomed.2015.10.003.

9. Kodner C. Common questions about the diagnosis and management of fibromyalgia. $\mathbf{A m}$ Fam Physician 2015; 91(7): 4728.

10. White KP, Speechley M, Harth $\mathrm{M}$, Ostbye T. Comparing selfreported function and work disability in 100 community cases of fibromyalgia syndrome versus controls in London, Ontario: the London Fibromyalgia Epidemiology Study. Arthritis Rheum 1999; 42(1): 76-83. doi: 10. 1002/1529-0131.

11. Beck A, Breth G, Hays R, Miller C. Psychiatric disorders and functional disability in patients with fibromyalgia. Permanente $\boldsymbol{J}$ 2000; 4: 3-21

12. Lacasse A, Bourgault $P$, Choinière $M$. Fibromyalgiarelated costs and loss of productivity: a substantial societal burden. BMC Musculoskelet Disord 2016; 17(1): 168. doi: 10. 1186/s12891-016-1027-6.

13. Robinson RL, Jones ML. In search of pharmacoeconomic evaluations for fibromyalgia treatments: a review. Expert Opin Pharmaco ther 2006; 7(8): 1027 39. doi: 10.1517/14656566.7.8. 1027.

14. Arnold, L.M. Management of psychiatric comorbidity in fibromyalgia. Curr Psychiatry Rep 2006; 8(3): 241-5. doi: 10. 1007/s11920-006-0030-2.
15. Goldenberg DL. Fibromyalgia syndrome a decade later: what have we learned? Arch Intern Med 1999; 159(8): 777-85. doi: 10.1001/archinte.159.8.777.

16. Carville SF, Arendt-Nielsen S, Bliddal H, Blotman F, Branco JC, Buskila D, et al. EULAR evidence based recommendations for the management of fibromyalgia syndrome. Ann Rheum Dis 2008; 67(4): 536-41. doi: 10.1136/ard.2007.071522.

17. Klement A, Hauser W, Bruckle $\mathrm{W}$, Eidmann U, Felde E, Herrmann M, et al. General principles of therapy, coordination of medical care and patient education in fibromyalgia syndrome and chronic widespread pain. Schmerz 2008; 22(3): 28394. doi: 10.1007/s00482-0080673-5.

18. Vázquez-Rivera S, GonzálezBlanch C, Rodríguez-Moya L, Morón D, González-Vives S, Carrasco JL. Brief cognitivebehavioral therapy with fibromyalgia patients in routine care. Compr Psychiatry 2009; 50: 517-25. doi: $10.1016 / \mathrm{j}$. comppsych.2009.01.008.

19. Okifuji A, Gao J, Bokat C, Hare BD. Management of fibromyalgia syndrome in 2016. Pain Manag 2016; 6(4): 383-400. doi: 10.2217/pmt-2016-0006.

20. Pérez de la Cruz S, Lambeck J. A new approach to the improvement of quality of life in fibromyalgia: a pilot study on the effects of an aquatic Ai Chi program. Int $J$ Rheum Dis 2016 (in press). doi: 10.1111/1756-185x.12930.

21. Tran ST, Guite JW, Pantaleao A, Pfeiffer M, Myer GD, Sil S, et al. Preliminary outcomes of a crosssite cognitive-behavioral and neuromuscular integrative training intervention for juvenile fibromyalgia. Arthritis Care Res 2016; 69(3): 413-420. doi: 
10.1002/acr. 22946

22. Bernardy K, Füber N, Köllner V, Häuser W. Efficacy of cognitivebehavioral therapies in fibromyalgia syndrome- A systematic review and metaanalysis of randomized controlled trials. Rheumatol 2010; 37(10): 1991-2005. doi: 10.3899/jrheum. 100104.

23. King SJ, Wessel J, Bhambhani Y, Sholter D, Maksymowyc W. The effects of exercise and education, individually or combined, in women with fibromyalgia. Rheumatol 2002; 29(12): 26202627.

24. Luciano JV, Martínez N, Peñarrubia-María MT, Fernández-Vergel R, GarcíaCampayo J, Verduras C, et al. Effectiveness of a psychoeducational treatment program implemented in general practice for fibromyalgia patients: A randomized controlled trial. Clin J Pain 2011; 27(5): 383-91. doi: 10.1097/ajp.0b013e31820b1 31c.

25. Gowans SE, ADeHueck A, Voss $\mathrm{S}$, Richardson M. A randomized, controlled trial of exercise and education for individuals with fibromyalgia. Arthrit Care Res 1999; 12(2): 120-128. doi: 10.1002/1529-0131(199904)12:

2<120::aid-art7>3.0.co;2-4.

26. Keel PJ, Bodoky C, Gerhard U, Müller W. Comparison of integrated group therapy and group elaxation training for fibromyalgia. Clin J Pain 1998; 14(3): 232-8. doi: 10.1097/ 00002508-19980 9000-00010.

27. Kaplan KH, Goldenberg DL, Galvin-Nadeau M. The impact of a meditation-based stress reduction program on fibromyalgia. Gen Hosp Psychiatry 1993; 15(5): 284-89. doi: $\quad 10.1016 / 0163-8343(93)$ 90020-o.

28. Grossman P, Tiefenthaler-Gilmer U, Raysz U, Kesper U. Mindfulness training as an intervention for fibromyalgia: Evidence of postintervention and 3-year follow-up benefits in wellbeing. Psycho Ther Psychosom 2007; 76(4): 226-33. doi: 10 1159/000101501.

29. Khan Niazi A, Khan Niazi S. Mindfulness-based stress reduction: a non-pharmacological approach for chronic illnesses. $N$ Am J Med Sci 2011; 3(1): 20-23. doi: 10. 4297/najms.2011.320.

30. Woolfolk RL, Lehrer PM. Principles and practice of stress management. New York: The Guilford Press, 1984.

31. Forqanizade J, Abhari R, Shakibi MR, Pirozan M, Samadi F, Tavakoli- Bazaz S. Prevalence of rheumatoid in Tehran. J Iran Univers Med Sci 1995; 3: 182191.

32. Yunus M.B. Fibromyalgia syndrome: Blueprint for reliable diagnosis. Consultation 1994; 12 : 60-74.

33. Bernstein DA, Borkovec TD. Progressive relaxation training: a manual for the helping professions. Champaign, IL: Research Press, 1973.

34. Jacobson, E. Progressive relaxation. Chicago: University of Chicago Press, 1938.

35. Hawker GA, Mian S, Kendzerska T, French M. Measures of adult pain: Visual Analog Scale for Pain (VAS Pain), Numeric Rating Scale for Pain (NRS Pain), McGill Pain Questionnaire (MPQ), Short-Form McGill Pain Questionnaire (SF-MPQ), Chronic Pain Grade Scale (CPGS), Short Form-36 Bodily Pain Scale (SF-36 BPS), and Measure of Intermittent and Constant Osteoarthritis Pain (ICOAP). Arthritis Care Res 2011; 63(S11): S240-S252. doi: 10.1002/acr.20543.

36. Fadaizadeh L, Habib, E, Sami K. Comparison of visual analogue scale and faces rating in measuring acute postoperative pain. Arch Iranian Med 2009; 12(1): 73-5.

37. Lovibond PF, Lovibond SH. The structure of negative emotional states: Comparison of the Depression Anxiety Stress Scales (DASS) with the Beck Depression and Anxiety Inventories. Behav Res Ther 1995; 33(3): 335-43. doi: 10. 1016/0005-7967(94)00075-u.

38. Brown TA, Chorpita BF, Korotitsch W, Barlow DH. Psychometric properties of the Depression Anxiety Stress Scales (DASS) in clinical samples. Behav Res Ther 1997; 35(1): 79-
89. doi: 10.1016/s0005-7967(96) 00068-x.

39. Asghari A, Saed F, Dibajnia P. Psychometric properties of the Depression Anxiety Scales -21 (DASS-21) in a non-clinical Iranian sample. Int $\boldsymbol{J}$ Psychol 2008; 4: 82-102.

40. Fors EA, Sexton H, Gotestam KG. The effect of guided imagery and amitriptyline on daily fibromyalgia pain. Psychiatr Res 2002; 36(3): 179-87. doi: 10. 1016/s0022-3956(02)00003-1.

41. Menzies V, Kim S. Relaxation and guided imagery in Hispanic persons diagnosed with fibromyalgia: a pilot study. Fam Community Health 2008; 31(3): 204-12. doi: 10.1097/01.fch. 0000324477.48083.08.

42. Anderson FJ, Winkler AE. An Integrated model of group psychotherapy for Pa-tents with fibromyalgia. Int $\boldsymbol{J}$ Group Psychoth 2007; 57(4): 451-74. doi: 10.1521/ijgp.2007.57.4.451.

43. Castel A, Perez M, Sala J, Padrol A, Rull M. Effect of hypnotic suggestion on fibromyalgic pain: Comparison between hypnosis and relaxation. Europ J Pain 2007; 11(4): 463-68. doi: 10. 1016/j.ejpain.2006.06.006.

44. Wigers SH, Stiles TC, Vogel PA. Effects of aerobic exercise versus stress management treatment in fibromyalgia A 4.5 year prospective study. Scand $J$ Rheumatol 1996; 25(2): 77-86. doi: 10.3109/03009749609069212.

45. Staud R, Cannon RC, Mauderli AP, Robinson ME, Price DD, Vierck CJ Jr. Temporal summation of pain from mechanical stimulation of muscle tissue in normal controls and subjects with fibromyalgia syndrome. Pain 2003; 102(1): 87 95. doi: 10.1016/s0304-3959(02) 00344-5.

46. Van Houdenhove B, Egle U. Fibromyalgia: a stress disorder? Piecing the biopsychosocial puzzle together. Psycho Ther Psychosom 2004; 73(5): 267-75. doi: 10.1159/000078843.

47. Gold PW, Chrousos GP. Organisation of the stress system and its dysregulation in melancholic and atypical depression: high vs low CRH/NE states. Mol Psychiatry 2002, 7(3): 
Relaxation versus stress management training for fibromyalgia

254-75. doi: 10.1038/sj.mp 4001032 .

48. Van Houdenhove B: Listening to CFS. Why we should pay more attention to the story of the patients. J Psychosom Res 2002; 52(6): 495-99. doi: 10.1016/ s0022-3999(01)00297-5.

49. Kivimäki $M$, Leino-Arjas $P$, Virtanen M, Elovainio M, Keltikangas-Järvinen L, Puttonen S. Work stress and incidence of newly diagnosed fibromyalgia. A prospective cohort study. $\boldsymbol{J}$ Psychosom Res 2004; 57(5): 41722. doi: 10.1016/j.jpsychores. 2003.10.013.

50. Zautra AJ, Fasman R, Reich JW, Harakas P, Johnson LM, Olmsted
ME. Fibromyalgia: evidence for deficits in positive affect regulation. Psychosom Med 2005; 67(1): 147-155. doi: 10. 1097/01.psy.0000146328.52009.

23.

51. Karimian M, Kashefolhagh F, Dadashi MS, Chharbaghi Z. The effect of relaxation and mental imagery on self-efficacy, competitive anxiety and sportive performance. $\mathrm{Br}$ J Sports Med 2010; 44(Suppl 1): 57. doi: 10. 1136/bjsm.2010.078725.192.

52. Kim SD, Kim HS. Effects of a relaxation breathing exercise on anxiety, depression, and leukocyte in hemopoietic stem cell transplantation patients.
Cancer Nursing 2005; 28(1): 79 83. doi: 10.1097/00002820200501000-00012.

53. Johansson N. Effectiveness of a stress management program in reducing anxiety and depression in nursing students. $\boldsymbol{J}$ Am Coll Health 1991; 40(3): 125-9. doi: 10. 1080/07448481.1991.9936268.

54. Burckhardt CS, Mannerkorpi K, Hedenberg L, Bjelle A. A randomized, controlled clinical trial of education and physical training for women with fibromyalgia. Rheumatol 1994; 21(4): 714-20. 